\title{
Myoelectric activity imaging and decoding with multichannel surface EMG for enhanced everyday life applicability
}

\author{
Fiorenzo Artoni, Stefan Kreipe, Member, IEEE, and Silvestro Micera, Senior Member, IEEE
}

\begin{abstract}
Surface electromyography (sEMG) has been increasingly used to evaluate neurophysiological aspects of movements, especially for rehabilitation. The traditional musclespecific sEMG approach requires considerable expertise to correctly position bipolar derivations over each muscle of interest. This reduced the availability of myoelectric imaging tools for non-professional users in everyday life conditions. High-density EMG (HDsEMG) consists of recording the sEMG via a dense array of electrodes. The high number of channels required to perform proper HDsEMG however restricts the surface that can be imaged only to a narrow area, which limits the usability of the approach to few applications. Here we demonstrate the usability and advantages of a medium-density sEMG (MDsEMG). We recorded the muscle activity of a subject performing repeated arm flexion and extension simultaneously using both, muscle-specific SEMG (4 derivations over the biceps and triceps) and MDsEMG (28 monopolar derivations, positioned around the arm circumference). Additionally, the elbow angle was measured. Grand-average dynamic-timewarped amplitudes showed similar activation patterns for both modalities. However, MDsEMG allowed to extract activation maps that highlighted the spatial activation of muscles at different movement phases (namely minimum and maximum acceleration). MDsEMG also proved superior in decoding the elbow angle and allowed to map the regions of maximum significance. In conclusion, MDsEMG is a viable alternative to muscle-specific and HD sEMG. It does not require expertise in electrode positioning and is able to cover large surfaces. It thus can pave the way to easier myoelectric imaging in everyday life and more effective rehabilitation treatments.
\end{abstract}

\section{INTRODUCTION}

Surface electromyography (sEMG) is becoming increasingly important in many applications, including clinical/biomedical assessment, control of prostheses or rehabilitation devices, human machine interactions etc. The recording and analysis of myoelectric activity allows a more standardized and precise evaluation of the neurophysiological aspects of movement, in particular in rehabilitative and assistive contexts [1].

Despite the growing interest of the research community in myoelectric imaging and the promising results, limitations in

F. Artoni, S. Kreipe,and S. Micera are with the Bertarelli Foundation Chair in Translational Neuroengineering, Center for Neuroprosthetics and Institute of Bioengineering, École Polytechnique Fédérale de Lausanne (EPFL), 1202 Geneva, Switzerland (correspondence to: fiorenzo.artoni@epfl.ch)

F. Artoni and S. Micera are also with the Biorobotics Institute, Sant'Anna School of advanced studies, Pisa mobility and robustness of current EMG devices limit their use in everyday life situations $[2,3]$.

Current standard practice for measuring sEMG consists of carefully positioning for each muscle of interest, a pair of electrodes on top of the muscle plus often a reference on a neutral region (e.g. elbow) to form bipolar derivations $[4,5]$.

Correct positioning and labelling of the electrodes as well as selection of the muscles of interest for a particular application requires a certain degree of specialized knowledge, often available only to clinicians. This is a further barrier to the widespread use of myoelectrical imaging in in everyday contexts. On top of that, especially for myoelectric control of prostheses for upper limb amputees, low residual muscle innervation might render correct positioning very difficult, even to a specialized clinician [6].

Another setup called high-density EMG (HDsEMG), has been proposed by several authors [7] with the aim of partly overcoming the aforementioned issues. HDsEMG consists of measuring the EMG with a grid of electrodes, i.e., an ensemble of sensors that records spatially-correlated data. As showed by Afsharipour et al. [8], the inter-electrode distance (IED) needs to be smaller than $10 \mathrm{~mm}$ to avoid spatial aliasing effects. Plus, constraints on the number of channels that can be acquired, narrow the surface area that can actually be covered, limiting HDsEMG for everyday applications.

A trade-off solution is spreading a number of electrodes (32, 64 or 128 monopolar derivations - easily recorded by most compact amplifiers) on the skin, irrespectively to the muscle positions or application $[9,10]$ and with larger IED, to form a "medium density EMG" - MDsEMG.

Inzelberg et al. [11] used a similar method to acquire sEMG data on facial muscles to investigate human facial expressions in everyday life situations. They used the spatial information obtained to discriminate between different active muscles to identify the relevant muscles contributing to each expression. Also, they point out the advantage of the simple placement procedure. In [12] MDsEMG was used to achieve simultaneous, proportional, multi-axis prosthesis control. Spatial information obtained by means of an electrode grid

S. Kreipe is also with the Biomedical Engineering Laboratory, Institute for Biomedical Engineering and Computer Science, Technical University of Ilmenau

This work was supported by the National Competence Center in Research (NCCR) in Robotics. F. Artoni's contributions were also supported the European Union's Horizon 2020 research and innovation program under the H2020 Marie Skłodowska-Curie Actions Grant Agreement No. 750947. 

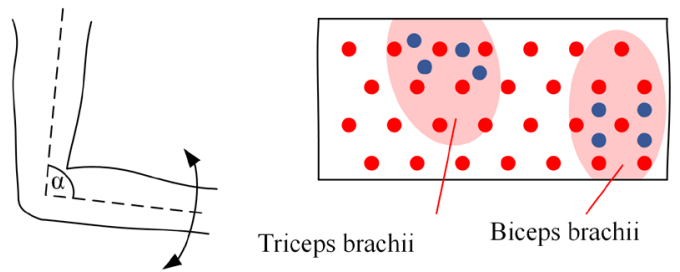

Figure 1.

Task and Electrode placement. The monopolar electrode grid is shown in red, the bipolar pairs in blue.

was used to decode hand and finger movements proportionally and with high accuracy.

In [13] MDsEMG activation maps were derived from forearms during wrist and finger movements. After reducing the data dimensionality using non-negative matrix Factorization, segmentation of the activation areas could be achieved. It was shown that for every finger there exists a special area on the forearm, where muscle activation can be measured during movement.

Despite the easy placement of electrodes, MDsEMG is still not widely used. The aim of this work is to demonstrate the usability and advantages of muscle-unspecific multichannel sEMG by means of an example experiment. We tested both muscle-specific (sEMG with four bipolar derivations) and muscle-unspecific MDsEMG by recording them simultaneously in one setup. The difference between the measurements was evaluated in terms of EMG envelopes and accuracy in movement- trajectory decoding.

Lastly, we computed the muscle activation maps and decoding accuracy maps to highlight the spatial information yielded by the multichannel approach.

\section{MethodS}

\section{A. Subject and Task}

The experiment was carried on one healthy, male subject who gave his written informed consent. The recordings were carried out in agreement with the Declaration of Helsinki. He was asked to flex and extend his forearm repeatedly without supination and pronation. The task was repeated 25 times per trial. Three trials were recorded, separated by 2-minute breaks to avoid fatigue.

\section{B. Experimental Setup}

Surface electromyogram (sEMG) was simultaneously recorded with two modalities. First, muscle-specific sEMG was measured by placing two pairs of bipolar channels respectively on the biceps brachii and triceps brachii. The signals were recorded with a wireless Noraxon system (Telemyo Desktop DTS). Muscle-unspecific multichannel sEMG (MDsEMG) was simultaneously measured around the whole upper arm via a total of 28 electrodes, placed in 4 rows around the circumference of the arm, each including 7 monopolar channels. The IED was around $30 \mathrm{~mm}$. The MDsEMG signals were recorded via an "ANT Neuro EEGO Mylab" EEG amplifier with reference and ground electrodes placed over the elbow bones. For both recording modalities, conventional disposable medium size $\mathrm{Ag} / \mathrm{AgCl}$ sEMG electrodes (spes medica DENIS01520) were used. Movement kinematics were recorded with a Vicon motion capture system. All measurements were offline synchronized by means of TTL pulses sent directly to all recording devices via a Labjack T7Pro as in [14]. The task and electrode positioning can be seen in Figure 1.

\section{Data Processing}

Elbow angles and angular accelerations were calculated from the motion capture data. Raw EMG signals, recorded at a sampling frequency of $2000 \mathrm{~Hz}$ (both bipolar and monopolar derivations) were high-pass filtered (zero-phase $15 \mathrm{~Hz}, 18 \mathrm{th}$ order, Butterworth) to ensure stationarity and remove movement artifacts [15], and low-pass filtered (zero-phase $300 \mathrm{~Hz}, 11$ th order, Butterworth) to remove high-frequency noise. EMG envelopes were computed by rectifying the filtered data and further applying a low-pass filter (zerophase, $10 \mathrm{~Hz}, 10$ th order, Butterworth) to smooth the data. To compute the activation maps we epoched the single sEMG derivation data, according to each movement repetition. To account for different lengths of each epoch we performed dynamic time warping [16]. We computed the grand average ( \pm standard deviation) over all movements and extracted the "activation map" (color-coded amplitudes, smoothed with a 2D spline interpolation) at the times of maximum and minimum accelerations.

\section{Movement decoding}

We also evaluated the performance in decoding the elbow angle. To this aim we performed a least-square regression on the movement trajectory (elbow angle) using either the 4 bipolar (sEMG) or 28 monopolar (MDsEMG) derivations. To extract the features for the decoding, we used $150 \mathrm{~ms}$
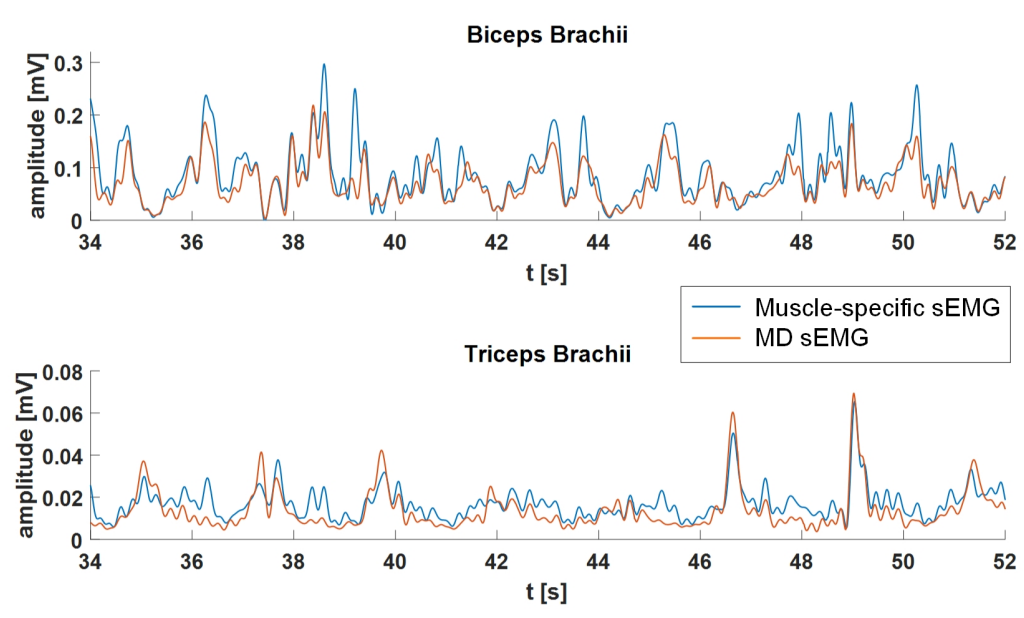

Figure 2. EMG envelopes of muscle-specifc channels and the nearest bipolar derivations of the MD sEMG grid on the biceps brachii and triceps brachii 


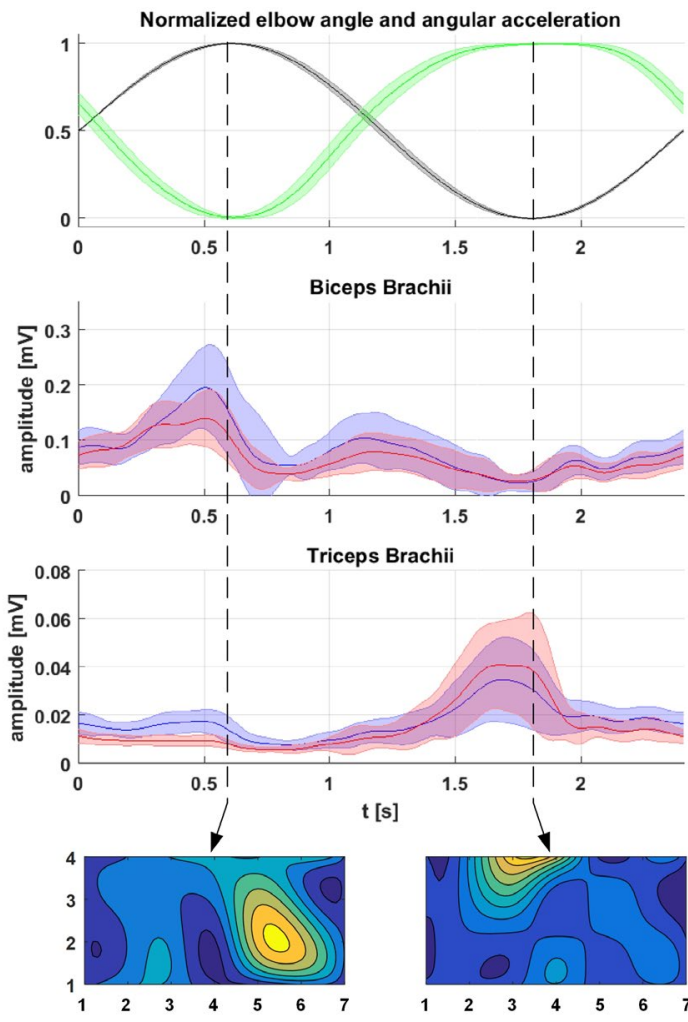

Figure 3. Average EMG envelopes per movement. The blue lines represent the muscle-specific EMG, the red ones the measurements by the electrode grid. The activation maps are shown for maximum and minimum acceleration. The axis indicate the electrode column and row.

windowing with $50 \%$ overlap. For each window the mean average value (MAV) and root mean square value (RMS) were extracted as simple features. We performed the regression using each single and bipolar sEMG derivation. We solved the linear regression model

$$
\operatorname{argmin}_{\hat{\theta}}|Y-X \hat{\theta}|_{2}
$$

where $\mathrm{Y}$ is the matrix containing the elbow angle over time, $\mathrm{X}$ the features and $\hat{\theta}$ the estimated model. $\hat{\theta}=X^{+} Y$ with $X^{+}$the pseudoinverse of $X$. The regression model was trained on $70 \%$ of the data and tested on the remaining $30 \%$.

To assess the performance of the various approaches the correlation coefficient (coc) between the real and estimated angles was computed

as

$$
\operatorname{coc}=\frac{C_{x \hat{x}}}{\sigma_{x} \cdot \sigma_{\hat{x}}}
$$

where $x_{i}$ is the real and $\hat{x}_{i}$ the estimated angle, $C_{x \hat{x}}$ the covariance and $\sigma_{x}, \sigma_{\hat{x}}$ the respective standard deviations.

To estimate the indepent information of each channel, first the coc was computed using only the channels signal. Then, the difference between one and the coc using all channels information except the regarded, was substracted. The results were plotted as a topographic map of relative information content.

\section{RESULTS}

Though placed irrespectively of the muscle positions, the EMG envelopes of bipolar derivations obtained from the multichannel grid (MDsEMG) show high resemblance to those of muscle-specific channels (Figure 2).

This is further confirmed by Figure 3: the grand-average EMG envelopes per movement are shown together with their standard deviation. It is possible to observe that both modalities highlight significant information on muscle activity. Thanks to the electrode grid, it was possible to extract the activation maps (Figure 3, bottom panels) that clearly highlight the muscle activity during different instances of the movement. The left side map shows the main activation of the Biceps, the right side one that of the Triceps.

Figure 4 highlights the results of the decoding of the elbow angle. It can be seen that both modalities are able to decode the movement with multichannel EMG performing significantly better than the other modality. The correlation coefficient was 0.66 for the muscle-specific EMG and 0.95 for the multichannel electrode grid.

The map of the relative information content of the channels (Figure 5) shows that the area pertaining to the triceps carries the highest information content.

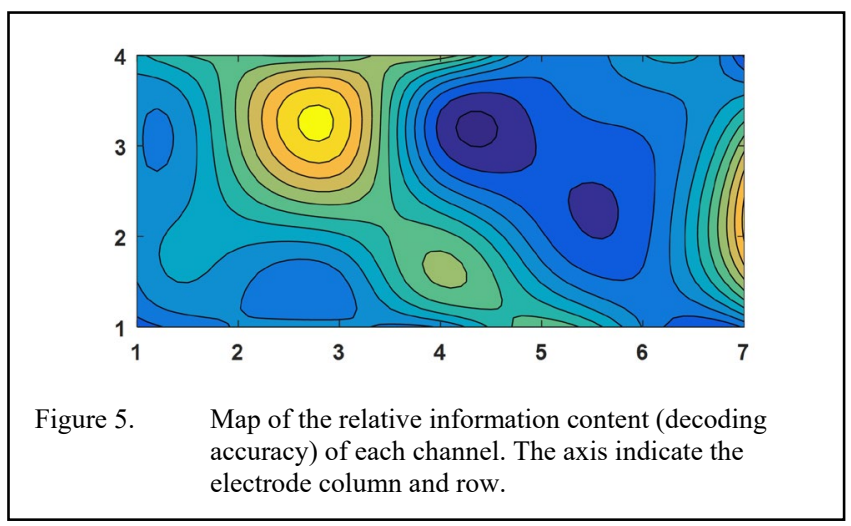

\section{DISCUSSION}

As it is possible to observe from Figures 2 and 3, there is no significant difference between the (averaged) EMG envelopes of muscle-specific sEMG and -unspecific MDsEMG measurements. Both modalities enabled to extract significant information and could therefore be used to measure muscle activity. In fact, as long as MDsEMG electrodes placement is sufficiently dense, bipolar derivations can always be reconstructed from monopolar ones.

The main advantage of multichannel EMG is however not only that of allowing the extraction of any number of bipolar derivations (depending on the number of electrodes), but also the possibility of dynamically visualizing spatial information on muscle activation, as shown in Figure 3 (bottom panels). This information has used for instance for facial electromyography [11], to better assess muscle fatigue [17] or even as feature to increase myoelectric control performance [7]. 


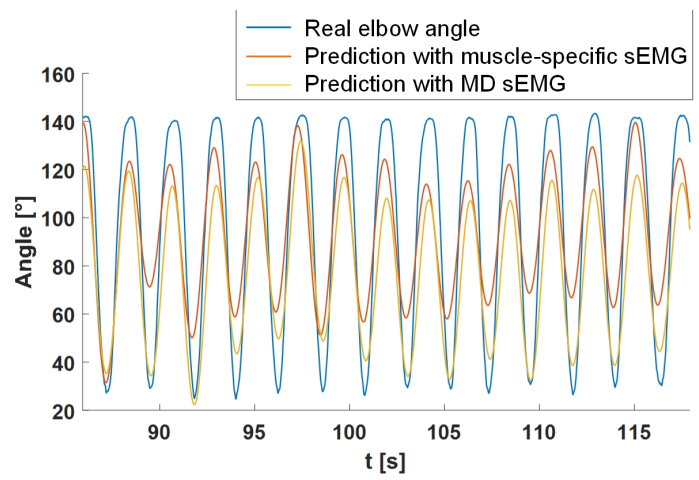

Figure 4. Prediction of the elbow angle based on the sEMG

Even without any spatial information (i.e. electrodes were not specifically placed over the muscle), as Figure 4 shows, the multichannel approach gives better results in decoding the elbow angle (regression approach).

The multichannel sEMG recording also enabled us to assess the information yielded by every channel (Figure 5). A possible explanation for the high information content of electrodes over the triceps, with respect to that of the biceps is likely due to the nature of the task, i.e., the biceps had a constantly higher activation level (Figure 3 ) than the triceps. This may have decreased the relative difference in the myoelectric activity during the task compared to the background signal, thereby effectively reducing the information level. Consequently, channels near the muscles but not directly on top of them might have had increased influence over the decoding performance.

The MDsEMG approach, with electrodes spread around the circumference of the arm yielded information not available with conventional muscle-specific sEMG. MDsEMG is thus very likely to enable better decoding of movement intentions for amputees, thus potentially allowing a better control of myoelectric prostheses. Though the MDsEMG setup required more materials available, it was easier and did not require any specific anatomical knowledge. With respect to HD sEMG approaches, a larger area was coverable. Therefore this approach paves the way to new opportunities concerning the standardization of EMG measurement setups and extends the possibility of muscle activity measurement also to nonprofessional users.

\section{CONCLUSION}

This work shows that multichannel sEMG is more than able to make up for muscle-unspecific electrode placement in terms of information yielded to the user. It can thus be considered as a valuable alternative to common musclespecific sEMG systems. Furthermore, the additional information gathered by the higher number of electrodes can be used to increase decoding accuracy, as well as robustness. However, the loss of information caused by spatial aliasing needs to be considered and quantified. Due to their numerous advantages, MDsEMG systems will potentially become more relevant in the future in everyday life applications or for increasing the performance of myoelectric prostheses for amputees. Further studies could reveal new methods on how to use the extra information to further increase the accuracy and robustness of multichannel-based EMG decoding.

\section{REFERENCES}

[1] R. H. Chowdhury, M. B. Reaz, M. A. B. M. Ali, A. A. Bakar, K Chellappan, and T. G. Chang, "Surface electromyography signal processing and classification techniques," Sensors, vol. 13, no. 9, pp. 12431-12466, 2013.

[2] N. Jiang, S. Dosen, K.-R. Muller, and D. Farina, "Myoelectric control of artificial limbs - is there a need to change focus?[In the spotlight]," IEEE Signal Processing Magazine, vol. 29, no. 5, pp. $152-150,2012$

[3] M. Atzori and H. Müller, "Control capabilities of myoelectric robotic prostheses by hand amputees: a scientific research and market overview," Frontiers in systems neuroscience, vol. 9, p. 162, 2015.

[4] M. Khezri and M. Jahed, "Real-time intelligent pattern recognition algorithm for surface EMG signals," Biomedical engineering online, vol. 6 , no. 1, p. $45,2007$.

[5] Y. Zhang et al., "Non-uniform Sample Assignment in Training Set Improving Recognition of Hand Gestures Dominated with Similar Muscle Activities," Frontiers in Neurorobotics, vol. 12, p. 3, 2018.

[6] M. A. Oskoei and H. Hu, "Myoelectric control systems-A survey," Biomedical Signal Processing and Control, vol. 2, no. 4, pp. 275 294, 2007.

[7] A. Stango, F. Negro, and D. Farina, "Spatial correlation of high density EMG signals provides features robust to electrode number and shift in pattern recognition for myocontrol," IEEE Transactions on Neural Systems and Rehabilitation Engineering, vol. 23, no. 2, pp. 189-198, 2015.

[8] B. Afsharipour, K. Ullah and R. Merletti, "Amplitude indicators and spatial aliasing in high density surface electromyography recordings," Biomedical Signal Processing and Control, pp. 170$179,2015$.

[9] M. AbdelMaseeh, T.-W. Chen, and D. W. Stashuk, "Extraction and classification of multichannel electromyographic activation trajectories for hand movement recognition," IEEE Transactions on Neural Systems and Rehabilitation Engineering, vol. 24, no. 6, pp. 662-673, 2016.

[10] Y. Fang, X. Zhu, and H. Liu, "Development of a surface emg acquisition system with novel electrodes configuration and signal representation," in International Conference on Intelligent Robotics and Applications, 2013, pp. 405-414: Springer.

[11] L. Inzelberg, D. Rand, S. Steinberg, M. David-Pur, and Y. Hanein, "A Wearable High-Resolution Facial Electromyography for Long Term Recordings in Freely Behaving Humans," Scientific reports, vol. 8, no. 1, p. 2058, 2018.

[12] D. Yatsenko, D. McDonnall, and K. S. Guillory, "Simultaneous, proportional, multi-axis prosthesis control using multichannel surface EMG," in Engineering in Medicine and Biology Society, 2007. EMBS 2007. 29th Annual International Conference of the IEEE, 2007, pp. 6133-6136: IEEE.

[13] M. Gazzoni, N. Celadon, D. Mastrapasqua, M. Paleari, V. Margaria and P. Ariano, "Quantifiying forearm muscle activity during wrist and finger movements by means of multi-channel electromyography," PloS one, vol 10, 2014.

[14] F. Artoni, A. Barsotti, E. Guanziroli, S. Micera, A. Landi, and F. Molteni, "Effective Synchronization of EEG and EMG for Mobile Brain/Body Imaging in Clinical Settings," Frontiers in Human Neuroscience, vol. 11, p. 652, 2017.

[15] F. Artoni, V. Monaco, and S. Micera, "Selecting the best number of synergies in gait: preliminary results on young and elderly people," IEEE Int Conf Rehabil Robot, vol. 2013, p. 6650416, Jun 2013.

[16] M. Müller, "Dynamic time warping," Information retrieval for music and motion, pp. 69-84, 2007.

[17] G. Marco, B. Alberto, and V. Taian, "Surface EMG and muscle fatigue: multi-channel approaches to the study of myoelectric manifestations of muscle fatigue," Physiological measurement, vol. 38, no. 5, p. R27, 2017. 